\title{
Gilles BOURQUE
}

Professeur de sociologie à l'Université du Québec à Montréal

(1984)

\section{"La fatigue \\ est dans tous les camps"}

Un document produit en version numérique par Jean-Marie Tremblay, bénévole, professeur de sociologie au Cégep de Chicoutimi

Courriel: jimt sociologue@,videotron.ca

Site web: http://www.uqac.ca/jmt-sociologue/

Dans le cadre de la collection: "Les classiques des sciences sociales"

Site web: http://www.uqac.ca/Classiques_des_sciences_sociales/

Une collection développée en collaboration avec la Bibliothèque

Paul-Émile-Boulet de l'Université du Québec à Chicoutimi

Site web: http://bibliotheque.uqac.uquebec.ca/index.htm 
Cette édition électronique a été réalisée par Jean-Marie Tremblay, bénévole, professeur de sociologie au Cégep de Chicoutimi à partir de :

Gilles Bourque, "La fatigue est dans tous les camps" in revue Conjoncture politique au Québec, no 5, printemps 1984, pp. 161 à 165. Dossier: Enseigner. Montréal : Éditions Albert Saint-Martin.

Professeur de sociologie à l'Université du Québec à Montréal, Gilles Bourque a été membre du comité de rédaction de "Parti pris" et directeur de la revue "Socialisme québécois". Il est l'auteur de plusieurs ouvrages, dont "L'État capitaliste et la question nationale".

Le 11 juillet 2004, M. Gilles Bourque nous autorisait à diffuser, dans Les Classiques des sciences sociales, toutes ses publications. Avec notre plus grand reconnaissance. JMT.

Courriel : bourque.gilles@uqam.ca

Polices de caractères utilisée :

Pour le texte: Times, 12 points.

Pour les citations : Times 10 points.

Pour les notes de bas de page : Times, 10 points.

Édition électronique réalisée avec le traitement de textes Microsoft Word 2001 pour Macintosh.

Mise en page sur papier format

LETTRE (US letter), 8.5' x 11',)

Édition complétée le 13 mai 2005 à Chicoutimi, Ville de Saguenay, province de Québec. 
Gilles Bourque,

\section{"La fatigue est dans tous les camps"}

\section{CONJONCTURE}

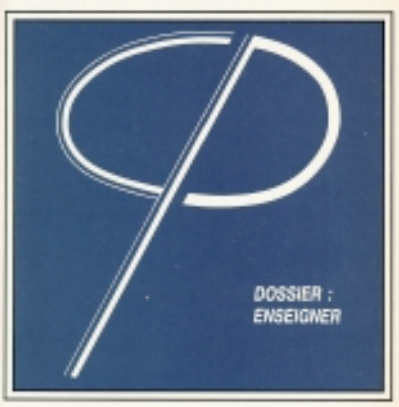

conjoncture politique au québec

in revue Conjoncture politique au Québec, no 5, printemps 1984, pp. 161 à 165. Dossier : Enseigner. Montréal : Éditions Albert Saint-Martin. 


\section{Gilles Bourque,}

\section{"La fatigue est dans tous les camps"}

in revue Conjoncture politique au Québec, no 5, printemps 1984, pp. 161 à 165. Dossier : Enseigner. Montréal : Éditions Albert Saint-Martin.

Dans la dernière livraison de Spirale, Gordon Lefebvre, qui nous avait habitué à du meilleur, se livre à une attaque tous azimuts contre les méchants universitaires qui ont participé récemment à la publication d'un livre intitulé Espace régional et nation. Il me fait l'insigne honneur de me présenter comme le maître d'œuvre de ce «discours idéologique braqué sur des fins inavouables». J'aurais, semble-t-il, ordonné à mes troupes de choc de braquer leurs armes sans laisser soupçonner leur véritable cible. Bref, nous n'en serions plus à la lutte des classes dans la théorie, mais à la guerre idéologique dans un terrain vague. Heureusement notre critique entend lever le voile en indiquant qui sont les véritables amis du peuple.

Gordon Lefebvre croit lire dans les textes de ce recueil «les arguments de la fatigue » laissant soupçonner (le mot est faible) que les auteurs se prépareraient à passer dans le camp des fédéralistes. La critique de Gordon Lefebvre fonctionne à l'amalgame. Elle tient du procès d'intention. Elle interpelle, au sens policier du terme, plutôt que d'ouvrir au débat. Sa critique ne questionne pas, elle accuse ou plutôt elle s'interroge sur les intentions animant les auteurs d'une production dont les résultats ne sont pas analysés sérieusement, mais condamnés sans autre forme de procès. Les arguments de son texte sont simples : 1 . L'introduction de la question régionale vise à voiler la question nationale. 2. Cette problématique ne peut être «qu'Ottawacentriste », c'est-à-dire «trudeauiste». 3. Les auteurs ne peuvent avoir que de mauvaises intentions. Nous oscillons ainsi entre les «intentions inavouables » et le sens caché enfin révélé. Si les auteurs sont fatigués, autant dire que la fatigue est aussi dans le camp de la critique. Dans les quelques lignes qui suivent, nécessairement trop brèves, j'aimerais d'abord rétablir certains faits élémentaires, la démarche insinuante de Gordon Lefebvre m'y obligeant, avant d'indiquer dans quel sens l'introduction de 
la notion de question régionale me semble utile pour comprendre et transformer la réalité québécoise.

Au début de sa critique Gordon Lefebvre oppose de façon manichéenne le livre, Au-delà du Parti québécois, du Centre de formation populaire et «l'ouvrage publié sous l'égide de Gilles Bourque », Espace régional et nation. Le premier, nous dit-il, « marque un effort louable... d'une essentielle prise en charge du problème national à gauche ». Mais malheureusement, ajoute-t-il plus loin, «le travail de réflexion entrepris par le CFP, l'influence trop restreinte et les faibles moyens dont dispose cette institution-carrefour populaire ne font pas le poids ». Ils ne font pas le poids devant « le prestige des auteurs (ceux d'Espace régional et nation) couplé aux moyens des diffuseurs ». Cette horrible alliance, se lamente Gordon Lefebvre, permettra de réaliser «ce coup (qui) vient parachever l'arasement des concepts et l'épandage de notions savatées auxquels le mouvement marxiste léniniste nous avait habités avant sa disparition ». Tout est là : les purs démunis de moyens, face aux esprits retors largement diffusés. On croirait assister à une lutte opposant les petits boutiquiers aux multinationales. On peut se demander en quoi publier chez Boréal Express assure une diffusion plus importante que chez Nouvelle Optique! Mais passons au plus équivoque. Gordon Lefebvre, critique sérieux, a sans nul doute lu intégralement les deux livres qu'il oppose. Si tel est bien le cas, je m'étonne qu'il ne se soit pas interrogé davantage sur le fait que l'un des protagonistes de son épopée, et pas n'importe lequel puisqu'il est présenté comme le chef des méchants, ait signé un texte dans chacun de ces ouvrages apparemment si diamétralement opposés. Deux réponses sont possibles : ou Gordon Lefebvre a refoulé dans son inconscient un élément du « dossier » qui ne cadrait manifestement pas avec ses thèses, ou, au contraire, il a pensé qu'il s'agissait là de la preuve suprême de la duplicité machiavélique de ces universitaires opportunistes et véreux.

Je soumets aux lecteurs les faits suivants. J'ai été membre à part entière du comité sur la question nationale du CFP et, à ce titre, j'ai discuté de la plupart des textes réunis dans le recueil Au-delà du Parti québécois. Je souscris entièrement à l'introduction du recueil que Gordon Lefebvre cite avec tant d'emphase, bien que je n'aie pas directement travaillé à sa rédaction puisqu'elle a été rédigée au moment où j'avais quitté le CFP pour participer à la formation du Mouvement socialiste (socialiste et indépendantiste, en passant !).

Je tiens à souligner de plus, n'ayant nullement l'intention de m'attribuer indûment les fruits d'un travail que je n'ai pas réalisé, que le livre Espace régional et nation n'a pas été publié «sous mon égide ». Cette publication résulte de l'initiative de Gérard Boismenu, Roch Denis, Lizette Jalbert et Daniel Salée. C'est à leur demande que Jules Duchastel et moi avons rédigé un texte qui fait état de la problématique que nous mettons en oeuvre dans le cadre de notre recherche sur le Duplessisme. Nous avons accepté de le faire parce que certaines préoccupations des auteurs rejoignaient les nôtres et que leur projet, ouvert aux débats dans sa facture même, laissait place à une multiplicité d'approches. 
Prenant encore une fois pour acquis que Gordon Lefebvre a lu les textes que j'ai signés dans les deux recueils, je comprends encore plus mal qu'il n'ait pas vu l'étroite filiation qui existe entre les deux approches. Il aurait sans doute compris que l'introduction de la question régionale n'est pas une nouveauté, mais qu'elle est présente dans les textes que je produis depuis plusieurs années (voir Bourque-Legaré, Le Québec la question nationale et Bourque-Dostaler, Socialisme et indépendance). On peut d'ailleurs en dire autant de la plupart des auteurs du recueil et de plusieurs autres auteurs(es) québécois(es). La nouveauté du recueil était précisément sa volonté d'initier un débat sur cette «fameuse » question régionale introduite (avec ou sans son concept) dans plusieurs productions québécoises ces dernières années. C'est à ce titre du moins que Jules Duchastel et moi avons accepté d'y participer.

Ceci dit, et pour quitter le simple terrain de la polémique, la facture même du recueil prête peut-être à ambiguïté. On a choisi de lancer le débat en réunissant des textes utilisant la notion de question régionale sans qu'au moins l'un d'entre eux ne porte spécifiquement sur l'analyse de la notion elle-même et les implications de son utilisation. Il s'agit donc d'un recueil qui appelle un débat, mais ne l'initie pas au sens strict. Au contraire, on peut dire que les cinq textes fonctionnent parallèlement et, pour certains d'entre eux, développent des positions relativement divergentes.

Tout en rappelant que cette faille, si faille il y a, ne mérite nullement un tel procès, je terminerai en indiquant dans quel sens cette notion me semble utile pour comprendre le Québec actuel.

Gordon Lefebvre reprend à son compte et sans nuance une thèse de Gérard Bergeron selon laquelle « il y a deux façons de traiter des problèmes constitutionnels au Canada : le schéma Ottawa-Centriste, le schéma Québec-Centriste ». S'il s'agit bien là d'une manière pertinente de saisir les deux principaux courants de la pensée québécoise, cette thèse est en même temps le constat d'un relatif blocage de l'analyse. Pour schématiser grossièrement, disons que la perspective «Ottawa-Centriste » s'attache à décrire l'évolution du fédéralisme canadien en considérant le Québec comme une région politique au sein de laquelle, dans le meilleur des cas, on reconnaîtrait une certaine forme de particularisme (national), tandis que le schéma «Québec-Centriste » tend à poser le Québec dans un rapport quasi univoque avec le Canada comme si nous avions affaire à deux blocs monolithiques homogènes et qui surtout se développaient en presque parfaite extériorité. Or, bien que ces deux approches aient permis de produire des analyses souvent pertinentes, de FaucherLamontagne à Marcel Rioux, il ne me semble nullement scandaleux de tenter de faire avancer les débats en faisant éclater l'enfermement relatif que l'une et l'autre perspective imposent à l'analyse. Avec beaucoup d'autres, j'ai d'abord tenté de déconstruire le schéma «Québec-Centriste» en montrant que des notions comme celles de société globale ou de classe ethnique, que certaines utilisations globalistes de la notion de nation ne rendaient pas compte de la pluralité et de la complexité de la réalité nationale québécoise. Pour ne prendre qu'un seul exemple, j'ai toujours insisté 
sur les contradictions qui traversaient le mouvement national québécois de l'époque des Patriotes, en passant par le Duplessisme, jusqu'à la récente prise en charge de ce mouvement par le Parti québécois. Que ce travail, chez moi comme chez d'autres, ait pu risquer, à un moment ou à un autre, de jeter le bébé avec l'eau du bain, nul n'en est plus conscient. Mais il m'a paru essentiel de prendre ce risque et je demeure toujours convaincu que seule une telle démarche permet «l'essentielle prise en charge du problème national à gauche » et «la recherche d'une réponse populaire, c'est-à-dire socialiste, démocratique, indépendantiste à la question nationale ».

Cette démarche m'a aussi amené à me rendre compte que le globalisme inhérent au «schéma Québec-Centriste» appelait et impliquait d'une certaine façon la perspective «Ottawa-Centriste» comme son envers nécessaire. Le globalisme inhérent au «schéma Québec-Centriste » est double: il oppose le Québec, société homogène, au Canada, terre indifférenciée. On pense, dans sa version limite, aux thèses présentant le Québec comme une colonie du Canada et à l'image du colonisé québécois face au colonisateur canadien. Il n'est donc pas étonnant qu'il ait été opposé à cette problématique un «schéma Ottawa-Centriste» s'attachant à décrire la complexité d'une réalité canadienne traversée par le régionalisme économique et politique. Nous connaissons tous les conséquences ultimes d'une telle approche qui conduit à traiter la réalité nationale québécoise comme un simple particularisme.

Nous assistons ainsi à un dialogue de sourds, la région étant opposée à la nation, la mosaïque à la ceinture fléchée qui finit par tenir de la pure et simple caricature. Toute utilisation de notions comme celles d'espace régional est dès lors reçue de façon soupçonneuse et accusatrice. Or, disons-le tout net et sans détour, l'apparition relativement récente de la notion d'espace ou de question régionales dans la littérature québécoise dite de gauche révèle du pire et du meilleur. Elle peut effectivement n'être qu'une reprise sous une forme à peine transformée du «schéma Ottawa-Centriste ». La question nationale, la réalité nationale québécoise, est alors littéralement réduite à celle d'un particularisme régional canadien. Il semble malheureusement nécessaire de rappeler ici que telle n'a jamais été la perspective que Jules Duchastel et moi avons développée dans l'article que nous avons signé dans ce recueil. Qu'en est-il donc?

Je partirai d'un énoncé en apparence trivial : le Québec fait partie du Canada ! Je rappellerai à Gordon Lefebvre que la perspective culturaliste qu'il semble vouloir défendre contre vents et marées n'a jamais pris sérieusement en considération cette réalité que nous vivons quotidiennement. Au mieux oppose-t-on la culture et la société globale « d'ici » à un Canada assimilateur et oppressif. Or la culture dont il est question, la réalité nationale québécoise, ne peut être comprise dans sa profondeur que dans son rapport politique au Canada. La province de Québec est une création politique de l'État canadien. La nation québécoise s'est créée dans le refoulement de la nation canadienne-française. Pour comprendre le Québec, la réalité de l'oppression nationale et la spécificité de la nation québécoise, on ne peut s'empêcher d'étudier la réalité de l'État canadien et le mode d'insertion du Québec dans l'ensemble fédéral. C'est dire qu'il importe de développer une approche à la fois politique et social- 
historique. La réalité nationale québécoise résulte des luttes socialement multiformes contre l'oppression nationale, luttes qui depuis la Proclamation royale de 1763 ont pour enjeu et pour objectif la reconnaissance politique de la réalité d'une communauté nationale distincte. Ces luttes n'ont jamais pu contrer décisivement l'oppression nationale, en 1774, en 1791, en 1840 comme en 1867.

Pour comprendre le Québec actuel, il est donc absolument indispensable d'étudier le fédéralisme canadien. Il ne s'agit nullement d'une question académique, mais d'une nécessité impérieuse pour le développement de toute pratique indépendantiste conséquente. Le texte que j'ai signé avec Jules Duchastel s'inscrit précisément dans cette perspective. Nous avons soutenu que la formation d'espaces politiques régionaux au Canada résultait d'un compromis politique imposé par les forces sociales dominantes dans les différentes colonies du British North America s'appuyant sur des communautés distinctes. La question régionale au Canada, le régionalisme politique provincial, constitue un mode de résolution des contradictions au sein de l'ensemble sociétal canadien, ensemble qui n'a pu au départ fondre les différentes communautés historiques qu'il intégrait et qui par la suite a dû se résoudre à fonctionner dans la division politique relative. En ce sens, l'espace politique provincial québécois se présente sous une double forme; son existence et sa reproduction résultent des luttes pour la reconnaissance de la communauté nationale, en même temps que sa réalité même, le provincialisme politique, traduit la volonté de son refoulement et de sa marginalisation. Dans le fédéralisme canadien, la question nationale québécoise est ravalée au niveau d'une simple question régionale : le Québec n'y est pas différent de l'Alberta ou de l'Île du Prince-Edouard.

Ceci dit, il importe de ne pas sous estimer cette réalité du régionalisme canadien qui dépasse la seule question du Québec. La notion de question régionale ouvre à la compréhension de la particularité et de la précarité de l'État canadien. Elle permet d'aborder et d'éclairer la complexité des luttes politiques dans ce pays en nous débarrassant une fois pour toutes d'une vision simpliste et monolithique du Canada anglais. Elle permet de poser les luttes nationales dans leur rapport à une géopolitique dont la compréhension est essentielle, puisque, rappelons-le en terminant, le Québec actuel en est le produit. Us événements entourant le rapatriement de la constitution ont montré la complexité de la réalité politique canadienne aussi bien que les « vertus » et les limites relatives des questions régionales et nationales : en même temps que l'isolement du Québec souligne d'un trait rouge la différence essentielle entre ces deux questions, la dénaturation du projet initial de Pierre Elliott-Trudeau, sous la pression des autres provinces, indique comment le régionalisme politique pèse lourdement sur l'État canadien dans son ensemble.

Fin. 